\title{
LOW FREQUENCY ELECTROMAGNETIC SCATTERING THEORY FOR A MULTI-LAYERED CHIRAL OBSTACLE*
}

\author{
CHRISTODOULOS ATHANASIADIS ${ }^{\dagger}$ AND IOANNIS G. STRATIS ${ }^{\dagger}$
}

\begin{abstract}
We consider the problem of scattering of a plane electromagnetic wave by a multilayered chiral scatterer with a perfectly conducting core. We derive integral representations for the total exterior electric field, as well as for the electric far field pattern of this problem. Using the low frequency approximation, we reduce the scattering problem to an iterative sequence of problems in potential theory, and construct the leading term approximation of the electric far field pattern.
\end{abstract}

1. Introduction. The study of the scattering of low frequency electromagnetic waves by an obstacle was initiated by Lord Rayleigh in 1897. A method of obtaining succesive approximations in ascending powers of the wave number was described by Stevenson [19]. Significant contribution to the study of scattering in low frequencies has been made by Kleinman and by Jones (we refer to [13], [14] and [11], [12], respectively). Some important theorems for multiple scattering of electromagnetic waves were proved by Twersky [20]. The present authors [5] obtained low frequency solutions of the conductive problem for Maxwell's equations. Low frequency electromagnetic scattering theory has been developed by one of the authors [3] for a multi-layered scatterer. In [18], Picard investigates the limiting process as the angular frequency tends to zero for the solution of the classical Maxwell system. In [1], Ammari et al. obtain the limit of the scattered field solution of the Drude-Born-Fedorov equations as the frequency tends to zero, and show that the asymptotics depend on the topological properties of the considered domain. Low frequency expansions have also been used in acoustics and elasticity; see, for example, Dassios [8], Dassios and Kiriaki [9], and one of the authors [2].

A chiral object is a body that cannot be brought into congruence with its mirror image by translation and rotation. Chirality is common in a variety of naturally occurring and man-made objects (e.g., DNA in molecular scale, helices). From an operational point of view, chirality is introduced into the classical Maxwell equations by a pair of generalized constitutive relations in which electric and magnetic fields are coupled via a new material parameter. The monograph [16] by Lakhtakia contains a large part of work on scattering problems involving chiral media. Low frequency expansions in chiral media have been used by Lakhtakia et al. [17], Lakhtakia [15], and Jaggard et al. [10].

The purpose of this work is to explore the low frequency scattering problem for an electromagnetic plane wave incident upon a multi-layered chiral scatterer with a perfectly conducting core. In Section 2, we describe the multi-layered chiral scatterer and formulate the corresponding scattering problem for the electric field only. In Section 3, we construct an integral representation for the total exterior electric field in which the transmission and boundary conditions, along with the behaviour at infinity, are incorporated. Moreover, we derive the electric far field pattern of the considered problem. In Section 4, we reduce the scattering problem to a series of problems in potential theory which can be solved successively in terms of expansions in appropriate harmonic functions; we also construct the leading term approximation of the electric

\footnotetext{
${ }^{*}$ Received Febuary 4, 1998; revised Sept. 15, 1998.

†University of Athens, Department of Mathematics, Panepistimiopolis, GR-157 84 Athens, Greece (istratis@math.uoa.gr).
} 
far field pattern. Finally, Section 5 contains some remarks and comments on other related research work.

2. Formulation of the problem. The multi-layered chiral scatterer $\Omega$ is a bounded, convex, and closed subset of $\mathbb{R}^{3}$, with a $C^{2}$-boundary $S_{0}$. The interior of $\Omega$ is divided by means of closed and nonintersecting $C^{2}$-surfaces into subsets (layers) $\Omega_{j}$, with $\partial \Omega_{j} \cap \partial \Omega_{j+1}=S_{j}, j=1,2, \ldots, N$. The surface $S_{j-1}$ surrounds $S_{j}$ and there is one normal unit vector $\hat{\mathbf{n}}(\mathbf{r})$ at each point $\mathbf{r}$ of any surface $S_{j}$ pointing at $\Omega_{j}$. Each $\Omega_{j}, j=1,2, \ldots, N$, is occupied by a homogeneous isotropic chiral medium with electric permittivity $\varepsilon_{j}$, magnetic permeability $\mu_{j}$, chirality measure $\beta_{j}$, and vanishing conductivity. The space $\Omega_{N+1}$, within which lies the origin, is the core of the multi-layered chiral scatterer, and appropriate boundary conditions are satisfied on its surface $S_{N}$, that may be either the perfect conductor condition, or the dielectric conditions, or an impedance boundary condition [7].

The exterior $\Omega_{0}$ of the scatterer is an infinite homogeneous isotropic achiral medium with electric permittivity $\varepsilon_{0}$, magnetic permeability $\mu_{0}$, and vanishing conductivity.

We shall consider the scattering of time-harmonic electromagnetic plane waves by the multi-layered chiral scatterer $\Omega$. Let $\left(\mathbf{E}^{\text {inc }}, \mathbf{H}^{\text {inc }}\right)$ and $\left(\mathbf{E}^{\mathrm{sc}}, \mathbf{H}^{\mathrm{sc}}\right)$ be the incident and the scattered waves, respectively. $\left(\mathbf{E}^{\text {sc }}, \mathbf{H}^{\text {sc }}\right)$ satisfies the well-known Silver-Müller radiation condition [7]. The total exterior field $\left(\mathbf{E}_{0}, \mathbf{H}_{0}\right)$ in $\Omega_{0}$ is given by

$$
\begin{aligned}
\mathbf{E}_{0} & =\mathbf{E}^{\mathrm{sc}}+\mathbf{E}^{\mathrm{inc}}, \\
\mathbf{H}_{0} & =\mathbf{H}^{\mathrm{sc}}+\mathbf{H}^{\mathrm{inc}},
\end{aligned}
$$

and satisfies the Maxwell equations

$$
\begin{aligned}
\nabla \times \mathbf{E}_{0} & =i \omega \mu_{0} \mathbf{H}_{0}, \\
\nabla \times \mathbf{H}_{0} & =-i \omega \varepsilon_{0} \mathbf{E}_{0}
\end{aligned}
$$

where $\omega$ is the angular frequency. The total field $\left(\mathbf{E}_{j}, \mathbf{H}_{j}\right)$ in each chiral layer $\Omega_{j}$, $j=1,2, \ldots, N$, satisfies the equations

$$
\begin{aligned}
& \nabla \times \mathbf{E}_{j}=i \omega \mu_{j} \frac{\gamma_{j}^{2}}{k_{j}^{2}} \mathbf{H}_{j}+\beta_{j} \gamma_{j}^{2} \mathbf{E}_{j}, \\
& \nabla \times \mathbf{H}_{j}=-i \omega \varepsilon_{j} \frac{\gamma_{j}^{2}}{k_{j}^{2}} \mathbf{E}_{j}+\beta_{j} \gamma_{j}^{2} \mathbf{H}_{j}
\end{aligned}
$$

where

$$
\gamma_{j}^{2}=\frac{k_{j}^{2}}{1-k_{j}^{2} \beta_{j}^{2}}
$$

with

$$
k_{j}^{2}=\omega^{2} \varepsilon_{j} \mu_{j}
$$

The equations (2.5) and (2.6) are derived by the source-free Maxwell curl postulates

$$
\nabla \times \mathbf{E}_{j}=i \omega \mathbf{B}_{j}, \quad \nabla \times \mathbf{H}_{j}=-i \omega \mathbf{D}_{j}
$$


along with the Drude-Born-Fedorov constitutive relations

$$
\mathbf{D}_{j}=\varepsilon_{j}\left(\mathbf{E}_{j}+\beta_{j} \nabla \times \mathbf{E}_{j}\right), \quad \mathbf{B}_{j}=\mu_{j}\left(\mathbf{H}_{j}+\beta_{j} \nabla \times \mathbf{H}_{j}\right) .
$$

For the physical meaning of $\beta_{j}, \gamma_{j}, k_{j}$ and more details about the constitutive relations for chiral media, we refer to [16]. We note that any solution of (2.3)-(2.6) is divergence free.

In order to develop the low-frequency theory for the scattering by $\Omega$, we consider the decoupling of $\mathbf{E}_{j}$ and $\mathbf{H}_{j}, j=0,1, \ldots, N$ in (2.3)-(2.6), eliminating the magnetic field. The equation thus arising is [3], [16],

$$
\nabla \times \nabla \times \mathbf{E}_{j}=\gamma_{j}^{2} \mathbf{E}_{j}+2 \beta_{j} \gamma_{j}^{2} \nabla \times \mathbf{E}_{j} \quad \text { in } \Omega_{j}, \quad j=0,1, \ldots, N
$$

where $\beta_{0}=0$, and $\gamma_{0}=k_{0}=\omega\left(\varepsilon_{0} \mu_{0}\right)^{1 / 2}$ is the free-space wave number in $\Omega_{0}$, and in view of (2.8), we get

$$
k_{j}^{2}=\frac{\varepsilon_{j} \mu_{j}}{\varepsilon_{0} \mu_{0}} k_{0}^{2}, \quad j=0,1, \ldots, N .
$$

The scattered electric field $\mathbf{E}^{\text {sc }}$ satisfies the Silver-Müller radiation condition

$$
\lim _{r \rightarrow \infty}\left[\mathbf{r} \times \nabla \times \mathbf{E}^{\mathrm{sc}}(\mathbf{r})+i k_{0} r \mathbf{E}^{\mathrm{sc}}(\mathbf{r})\right]=\mathbf{0}
$$

uniformly in all directions.

On the surfaces $S_{j}, j=0,1, \ldots, N-1$, we have the transmission conditions

$$
\begin{aligned}
\hat{\mathbf{n}} \times \mathbf{E}_{j} & =\hat{\mathbf{n}} \times \mathbf{E}_{j+1} \\
\hat{\mathbf{n}} \times \nabla \times \mathbf{E}_{j} & =\frac{\varepsilon_{j+1}}{\varepsilon_{j}} \frac{\gamma_{j}^{2}}{\gamma_{j+1}^{2}} \hat{\mathbf{n}} \times \nabla \times \mathbf{E}_{j+1}+\gamma_{j}^{2}\left(\beta_{j}-\frac{\varepsilon_{j+1}}{\varepsilon_{j}} \beta_{j+1}\right) \hat{\mathbf{n}} \times \mathbf{E}_{j+1} .
\end{aligned}
$$

The relation (2.15) arises from the standard dielectric condition $\hat{\mathbf{n}} \times \mathbf{H}_{j}=\hat{\mathbf{n}} \times \mathbf{H}_{j+1}$ on $S_{j}$ by substituting the magnetic field from (2.5).

We consider that the core is a perfect conductor, that is

$$
\hat{\mathbf{n}} \times \mathbf{E}_{N}=\mathbf{0} \text {, on } S_{N} \text {. }
$$

The proof that the above transmission problem has a unique solution can be found in [6].

The corresponding problem, arising by eliminating the electric field, consists of the equations in $\Omega_{j}, j=1,2, \ldots, N$

$$
\nabla \times \nabla \times \mathbf{H}_{j}=\gamma_{j}^{2} \mathbf{H}_{j}+2 \beta_{j} \gamma_{j}^{2} \nabla \times \mathbf{H}_{j}
$$

the transmission conditions on $S_{j}, j=1,2, \ldots, N-1$

$$
\begin{aligned}
\hat{\mathbf{n}} \times \mathbf{H}_{j} & =\hat{\mathbf{n}} \times \mathbf{H}_{j+1}, \\
\hat{\mathbf{n}} \times \nabla \times \mathbf{H}_{j} & =\frac{\mu_{j+1}}{\mu_{j}} \frac{\gamma_{j}^{2}}{\gamma_{j+1}^{2}} \hat{\mathbf{n}} \times \nabla \times \mathbf{H}_{j+1}+\gamma_{j}^{2}\left(\beta_{j}-\frac{\mu_{j+1}}{\mu_{j}} \beta_{j+1}\right) \hat{\mathbf{n}} \times \mathbf{H}_{j+1},
\end{aligned}
$$

the boundary condition on $S_{N}$

$$
\hat{\mathbf{n}} \times \nabla \times \mathbf{H}_{N}-\beta_{N} \gamma_{N}^{2} \hat{\mathbf{n}} \times \mathbf{H}_{N}=\mathbf{0},
$$


and the radiation condition

$$
\lim _{r \rightarrow \infty}\left[\mathbf{r} \times \nabla \times \mathbf{H}^{\mathrm{sc}}(\mathbf{r})+i k_{0} r \mathbf{H}^{\mathrm{sc}}(\mathbf{r})\right]=\mathbf{0}, \quad \text { uniformly in all directions, }
$$

with $\beta_{0}=0$ and $\gamma_{0}=k_{0}$. This problem can be treated by an entirely analogous procedure to that of the following sections.

REMARK. Instead of being a perfect conductor, the core may be considered to be chiral and penetrable. In this case, the electric field $\mathbf{E}_{N+1}$, in the interior of the core $\Omega_{N+1}$, will satisfy (2.11) with $j=N+1$, and the transmission conditions (2.14), (2.15) for $j=N$, on $S_{N}$. This problem can be studied essentially as in Sections 3 and 4.

3. The exterior field. In this section, we construct an integral representation of the total exterior electric field in which all the transmission and boundary conditions are incorporated. In order to do this, we begin with the following integral representation for the electric field, [20], [3]:

$$
\begin{aligned}
& \mathbf{E}^{\mathrm{sc}}(\mathbf{r})=\int_{S_{0}}\left[\left(\nabla \times \mathbf{E}^{\mathrm{sc}}\left(\mathbf{r}^{\prime}\right)\right) \cdot\left(\hat{\mathbf{n}} \times \tilde{\Gamma}\left(\mathbf{r}, \mathbf{r}^{\prime}\right)\right)\right. \\
&\left.-\left(\hat{\mathbf{n}} \times \mathbf{E}^{\mathrm{sc}}\left(\mathbf{r}^{\prime}\right)\right) \cdot\left(\nabla_{\mathbf{r}^{\prime}} \times \tilde{\Gamma}\left(\mathbf{r}, \mathbf{r}^{\prime}\right)\right)\right] d s\left(\mathbf{r}^{\prime}\right), \quad \mathbf{r} \in \Omega_{0}
\end{aligned}
$$

where $\tilde{\Gamma}\left(\mathbf{r}, \mathbf{r}^{\prime}\right)$ is the free-space dyadic Green's function

$$
\tilde{\Gamma}\left(\mathbf{r}, \mathbf{r}^{\prime}\right)=-\left(\tilde{I}+k_{0}^{-2} \nabla \otimes \nabla\right) \frac{e^{i k o\left|\mathbf{r}-\mathbf{r}^{\prime}\right|}}{4 \pi\left|\mathbf{r}-\mathbf{r}^{\prime}\right|},
$$

with $\tilde{I}=\hat{\mathbf{e}}_{1} \otimes \hat{\mathbf{e}}_{1}+\hat{\mathbf{e}}_{2} \otimes \hat{\mathbf{e}}_{2}+\hat{\mathbf{e}}_{3} \otimes \hat{\mathbf{e}}_{3}$ the identity dyadic $\left(\hat{\mathbf{e}}_{j}, j=1,2,3\right.$, are the cartesian unit vectors). Inserting (2.1) in (3.1) and taking into account that $\mathbf{E}^{\text {inc }}$ is a solution of the equation (2.11) for $j=0$, we obtain

$$
\begin{aligned}
\mathbf{E}_{0}(\mathbf{r})=\mathbf{E}^{\mathrm{inc}}(\mathbf{r})+\int_{S_{0}}\left[\left(\nabla \times \mathbf{E}_{0}\left(\mathbf{r}^{\prime}\right)\right)\right. & \cdot\left(\hat{\mathbf{n}} \times \tilde{\Gamma}\left(\mathbf{r}, \mathbf{r}^{\prime}\right)\right) \\
& \left.-\left(\hat{\mathbf{n}} \times \mathbf{E}_{0}\left(\mathbf{r}^{\prime}\right)\right) \cdot\left(\nabla_{\mathbf{r}^{\prime}} \times \tilde{\Gamma}\left(\mathbf{r}, \mathbf{r}^{\prime}\right)\right)\right] d s\left(\mathbf{r}^{\prime}\right) .
\end{aligned}
$$

Using the transmission conditions on $S_{0}$, the relation (3.3) becomes

$$
\begin{aligned}
& \text { (3.4) } \mathbf{E}_{0}(\mathbf{r})=\mathbf{E}^{\mathrm{inc}}(\mathbf{r})-\frac{\varepsilon_{1} \gamma_{0}^{2}}{\varepsilon_{0} \gamma_{1}^{2}} \int_{S_{0}} \hat{\mathbf{n}} \cdot\left[\left(\nabla \times \mathbf{E}_{1}\left(\mathbf{r}^{\prime}\right)\right) \times \tilde{\Gamma}\left(\mathbf{r}, \mathbf{r}^{\prime}\right)\right] d s\left(\mathbf{r}^{\prime}\right) \\
& +\gamma_{0}^{2} \frac{\varepsilon_{1}}{\varepsilon_{0}} \beta_{1} \int_{S_{0}} \hat{\mathbf{n}} \cdot\left[\mathbf{E}_{1}\left(\mathbf{r}^{\prime}\right) \times \tilde{\Gamma}\left(\mathbf{r}, \mathbf{r}^{\prime}\right)\right] d s\left(\mathbf{r}^{\prime}\right)-\int_{S_{0}} \hat{\mathbf{n}} \cdot\left[\mathbf{E}_{1}\left(\mathbf{r}^{\prime}\right) \times\left(\nabla_{\mathbf{r}^{\prime}} \times \tilde{\Gamma}\left(\mathbf{r}, \mathbf{r}^{\prime}\right)\right)\right] d s\left(\mathbf{r}^{\prime}\right) .
\end{aligned}
$$

Now, applying successively the dyadic form of the divergence theorem and taking into account that $\mathbf{E}_{j}$ and $\tilde{\Gamma}$ are solutions of (2.11) in $\Omega_{j}, j=1,2, \ldots, N$, and $\Omega_{0}$ respectively, and introducing the transmission conditions (2.14), (2.15) and the boundary condition (2.16), we obtain that

$$
\mathbf{E}_{0}(\mathbf{r})=\mathbf{E}^{\text {inc }}(\mathbf{r})+\mathbf{E}^{\mathrm{sc}(\mathrm{c})}(\mathbf{r})+\mathbf{E}^{\mathrm{sc}(\mathrm{a})}(\mathbf{r})
$$

where $\mathbf{E}^{\mathrm{sc}(\mathbf{c})}$ and $\mathbf{E}^{\mathrm{sc}(\mathrm{a})}$ denote, respectively, the chiral and the achiral parts of the electric scattered field $\mathbf{E}^{\mathrm{sc}}$, and are given by

$$
4 \pi \mathbf{E}^{\mathrm{sc}(\mathrm{c})}(\mathbf{r})=-\frac{\varepsilon_{N}}{\varepsilon_{0}} \beta_{N}^{2} k_{0}^{2} \int_{S_{N}}\left(\nabla \times \mathbf{E}_{N}\left(\mathbf{r}^{\prime}\right)\right) \cdot\left(\hat{\mathbf{n}} \times \tilde{\Gamma}\left(\mathbf{r}, \mathbf{r}^{\prime}\right)\right) d s\left(\mathbf{r}^{\prime}\right)
$$




$$
\begin{aligned}
& -k_{0}^{2} \sum_{j=1}^{N} \frac{\varepsilon_{j}}{\varepsilon_{0}} \beta_{j}^{2} \int_{\Omega_{j}}\left(\nabla \times \mathbf{E}_{j}\left(\mathbf{r}^{\prime}\right)\right) \cdot\left(\nabla_{\mathbf{r}^{\prime}} \times \tilde{\Gamma}\left(\mathbf{r}, \mathbf{r}^{\prime}\right)\right) d v\left(\mathbf{r}^{\prime}\right) \\
& -k_{0}^{2} \sum_{j=1}^{N} \frac{\varepsilon_{j}}{\varepsilon_{0}} \beta_{j} \int_{\Omega_{j}}\left(\nabla \times \mathbf{E}_{j}\left(\mathbf{r}^{\prime}\right)\right) \cdot \tilde{\Gamma}\left(\mathbf{r}, \mathbf{r}^{\prime}\right) d v\left(\mathbf{r}^{\prime}\right) \\
& -k_{0}^{2} \sum_{j=1}^{N} \frac{\varepsilon_{j}}{\varepsilon_{0}} \beta_{j} \int_{\Omega_{j}} \mathbf{E}_{j}\left(\mathbf{r}^{\prime}\right) \cdot\left(\nabla_{\mathbf{r}^{\prime}} \times \tilde{\Gamma}\left(\mathbf{r}, \mathbf{r}^{\prime}\right)\right) d v\left(\mathbf{r}^{\prime}\right), \mathbf{r} \in \Omega_{0}
\end{aligned}
$$

and

$$
\begin{aligned}
4 \pi \mathbf{E}^{s c(a)}(\mathbf{r})= & \frac{\mu_{0}}{\mu_{N}} \int_{S_{N}}\left(\nabla \times \mathbf{E}_{N}\left(\mathbf{r}^{\prime}\right)\right) \cdot\left(\hat{\mathbf{n}} \times \tilde{\Gamma}\left(\mathbf{r}, \mathbf{r}^{\prime}\right)\right) d s\left(\mathbf{r}^{\prime}\right) \\
& +k_{0}^{2} \sum_{j=1}^{N}\left(1-\frac{\varepsilon_{j}}{\varepsilon_{0}}\right) \int_{\Omega_{j}} \mathbf{E}_{j}\left(\mathbf{r}^{\prime}\right) \cdot \tilde{\Gamma}\left(\mathbf{r}, \mathbf{r}^{\prime}\right) d v\left(\mathbf{r}^{\prime}\right) \\
& +\sum_{j=1}^{N}\left(\frac{\mu_{0}}{\mu_{j}}-1\right) \int_{\Omega_{j}}\left(\nabla \times \mathbf{E}_{j}\left(\mathbf{r}^{\prime}\right)\right) \cdot\left(\nabla_{\mathbf{r}^{\prime}} \times \tilde{\Gamma}\left(\mathbf{r}, \mathbf{r}^{\prime}\right)\right) d v\left(\mathbf{r}^{\prime}\right), \mathbf{r} \in \Omega_{0} .
\end{aligned}
$$

The surface integrals in (3.6), (3.7) express the effect of the core, while the volume integrals express the contribution of each layer to the exterior field.

The behavior of the scattered electric wave in the region of radiation is described by the electric far field pattern $\mathbf{E}_{\infty}(\hat{\mathbf{r}})$, which is defined [7] by the relation

$$
\mathbf{E}^{\mathrm{sc}}(\mathbf{r})=\mathbf{E}_{\infty}(\hat{\mathbf{r}}) h\left(k_{0} r\right)+O\left(\frac{1}{r^{2}}\right), \quad r \rightarrow \infty,
$$

uniformly in all directions where $h(x)=e^{i x} /(i x)$ is the zeroth-order spherical Hankel function of the first kind. Substituting the asymptotic forms, [3],

$$
\begin{aligned}
\tilde{\Gamma}\left(\mathbf{r}, \mathbf{r}^{\prime}\right) & =-\frac{i k_{0}}{4 \pi}(\tilde{I}-\hat{\mathbf{r}} \otimes \hat{\mathbf{r}}) h\left(k_{0} r\right) e^{-i k_{0} \hat{\mathbf{r}} \cdot \mathbf{r}^{\prime}}+O\left(\frac{1}{r^{2}}\right), \quad r \rightarrow \infty \\
\nabla_{\mathbf{r}^{\prime}} \times \tilde{\Gamma}\left(\mathbf{r}, \mathbf{r}^{\prime}\right) & =-\frac{k_{0}^{2}}{4 \pi}(\tilde{I} \times \hat{\mathbf{r}}) h\left(k_{0} r\right) e^{-i k_{0} \hat{\mathbf{r}} \cdot \mathbf{r}^{\prime}}+O\left(\frac{1}{r^{2}}\right), \quad r \rightarrow \infty
\end{aligned}
$$

in $(3.6),(3.7)$ and, in view of (3.8), we obtain

$$
\mathbf{E}_{\infty}(\hat{\mathbf{r}})=\mathbf{E}_{\infty}^{(c)}(\hat{\mathbf{r}})+\mathbf{E}_{\infty}^{(\mathrm{a})}(\hat{\mathbf{r}})
$$

where

$$
\begin{aligned}
4 \pi \mathbf{E}_{\infty}^{(\mathbf{c})}(\hat{\mathbf{r}})=i k_{0}^{3} \frac{\varepsilon_{N}}{\varepsilon_{0}} \beta_{N}^{2} \int_{S_{N}}\left(\nabla \times \mathbf{E}_{N}\left(\mathbf{r}^{\prime}\right)\right) \cdot(\hat{\mathbf{n}} \times(\tilde{I}-\hat{\mathbf{r}} \otimes \hat{\mathbf{r}})) e^{-i k_{0} \hat{\mathbf{r}} \cdot \mathbf{r}^{\prime}} d s\left(\mathbf{r}^{\prime}\right) \\
+k_{0}^{4} \sum_{j=1}^{N} \frac{\varepsilon_{j}}{\varepsilon_{0}} \beta_{j}^{2} \int_{\Omega_{j}}\left(\nabla \times \mathbf{E}_{j}\left(\mathbf{r}^{\prime}\right)\right) \cdot(\tilde{I} \times \hat{\mathbf{r}}) e^{-i k_{0} \hat{\mathbf{r}} \cdot \mathbf{r}^{\prime}} d v\left(\mathbf{r}^{\prime}\right) \\
+i k_{0}^{3} \sum_{j=1}^{N} \frac{\varepsilon_{j}}{\varepsilon_{0}} \beta_{j} \int_{\Omega_{j}}\left(\nabla \times \mathbf{E}_{j}\left(\mathbf{r}^{\prime}\right)\right) \cdot(\tilde{I}-\hat{\mathbf{r}} \otimes \hat{\mathbf{r}}) e^{-i k_{0} \hat{\mathbf{r}} \cdot \mathbf{r}^{\prime}} d v\left(\mathbf{r}^{\prime}\right) \\
+k_{0}^{4} \sum_{j=1}^{N} \frac{\varepsilon_{j}}{\varepsilon_{0}} \beta_{j} \int_{\Omega_{j}} \mathbf{E}_{j}\left(\mathbf{r}^{\prime}\right) \cdot(\tilde{I} \times \hat{\mathbf{r}}) e^{-i k_{0} \hat{\mathbf{r}} \cdot \mathbf{r}^{\prime}} d v\left(\mathbf{r}^{\prime}\right)
\end{aligned}
$$


and

$$
\begin{aligned}
4 \pi \mathbf{E}_{\infty}^{(\mathrm{a})}(\hat{\mathbf{r}})=- & i k_{0} \frac{\mu_{0}}{\mu_{N}} \int_{S_{N}}\left(\nabla \times \mathbf{E}_{N}\left(\mathbf{r}^{\prime}\right)\right) \cdot(\hat{\mathbf{n}} \times(\tilde{I}-\hat{\mathbf{r}} \otimes \hat{\mathbf{r}})) e^{-i k_{0} \hat{\mathbf{r}} \cdot \mathbf{r}^{\prime}} d s\left(\mathbf{r}^{\prime}\right) \\
& -i k_{0}^{3} \sum_{j=1}^{N}\left(1-\frac{\varepsilon_{j}}{\varepsilon_{0}}\right) \int_{\Omega_{j}} \mathbf{E}_{j}\left(\mathbf{r}^{\prime}\right) \cdot(\tilde{I}-\hat{\mathbf{r}} \otimes \hat{\mathbf{r}}) e^{-i k_{0} \hat{\mathbf{r}} \cdot \mathbf{r}^{\prime}} d v\left(\mathbf{r}^{\prime}\right) \\
& -k_{0}^{2} \sum_{j=1}^{N}\left(\frac{\mu_{0}}{\mu_{j}}-1\right) \int_{\Omega_{j}}\left(\nabla \times \mathbf{E}_{j}\left(\mathbf{r}^{\prime}\right)\right) \cdot(\tilde{I} \times \hat{\mathbf{r}}) e^{-i k_{0} \hat{\mathbf{r}} \cdot \mathbf{r}^{\prime}} d v\left(\mathbf{r}^{\prime}\right) .
\end{aligned}
$$

4. The low frequency approximation. We consider an incident plane wave $\mathbf{E}^{\mathrm{inc}}(\mathbf{r})=\mathbf{p} e^{i k_{0} \hat{\mathbf{d}} \cdot \mathbf{r}}$ where the unit vector $\hat{\mathbf{d}}$ describes the direction of propagation, the constant vector $\mathbf{p} \in \mathbb{R}^{3}$ gives the polarization, and are such that $\mathbf{p} \cdot \hat{\mathbf{d}}=0$. $\mathbf{E}^{\text {inc }}$ is analytic at $k_{0}=0$ and it can be expanded into the convergent power series

$$
\mathbf{E}^{\mathrm{inc}}(\mathbf{r})=\mathbf{p} \sum_{m=0}^{\infty} \frac{\left(i k_{0}\right)^{m}}{m !}(\hat{\mathbf{d}} \cdot \mathbf{r})^{m} .
$$

We assume the standard low frequency hypothesis $k_{0} a \ll 1$, where $a$ is the radius of the smallest circumscribable sphere around the scatterer, [3], [8], [9], [13], [14]. We consider the following expansion of the fields $\mathbf{E}_{j}$ into convergent power series of $k_{0}$ :

$$
\mathbf{E}_{j}(\mathbf{r})=\sum_{m=0}^{\infty} \frac{\left(i k_{0}\right)^{m}}{m !} \Phi_{m}^{(j)}(\mathbf{r})
$$

where $\Phi_{m}^{(j)}(\mathbf{r})$ are independent of $k_{0}$. For the justification of this expansion, see the Appendix. Inserting the expansion (4.2) into (2.11) and equating equal powers of $k_{0}$, we obtain for $j=0,1, \ldots, N, m=0,1,2, \ldots$, that

$$
\begin{aligned}
\nabla & \times \nabla \times \Phi_{m}^{(j)}(\mathbf{r}) \\
& =m(m-1) \frac{\varepsilon_{j} \mu_{j}}{\varepsilon_{0} \mu_{0}}\left[\beta_{j} \nabla \times \nabla \times \Phi_{m-2}^{(j)}(\mathbf{r})+2 \beta_{j} \nabla \times \Phi_{m-2}^{(j)}(\mathbf{r})+\Phi_{m-2}^{(j)}(\mathbf{r})\right]
\end{aligned}
$$

Let us note that $\nabla \cdot \mathbf{E}=0$ renders $\nabla \cdot \Phi_{m}^{(j)}=0$. In view of this relation, (4.3) becomes the Laplace equation for $m=0,1$, and the Poisson equation for $m \geq 2$; in the latter case, the right-hand side of (4.3) is dependent on the $(m-2)$-th order coefficient which is known from previous steps.

The boundary conditions (2.14) and (2.15) are transformed into

$$
\begin{aligned}
& \hat{\mathbf{n}} \times \Phi_{m}^{(j)}(\mathbf{r})=\hat{\mathbf{n}} \times \Phi_{m}^{(j+1)}(\mathbf{r}) \\
& \hat{\mathbf{n}} \times \nabla \times \Phi_{m}^{(j)}(\mathbf{r})=\frac{\mu_{j}}{\mu_{j+1}} \hat{\mathbf{n}} \times \nabla \times \Phi_{m}^{(j+1)}(\mathbf{r})+\frac{m(m-1)}{\varepsilon_{0} \mu_{0}}\left\{\beta_{j}^{2} \varepsilon_{j} \mu_{j} \hat{\mathbf{n}} \times \nabla \times \Phi_{m-2}^{(j)}(\mathbf{r})\right.
\end{aligned}
$$

$$
\left.-\beta_{j+1}^{2} \varepsilon_{j+1} \mu_{j+1} \hat{\mathbf{n}} \times \nabla \times \Phi_{m-2}^{(j+1)}(\mathbf{r})+\varepsilon_{j} \mu_{j}\left(\beta_{j}-\frac{\varepsilon_{j+1}}{\varepsilon_{j}}\right) \hat{\mathbf{n}} \times \Phi_{m-2}^{(j+1)}(\mathbf{r})\right\},
$$

on $S_{j}, j=0,1, \ldots, N-1$, and the boundary condition (2.16) becomes

$$
\hat{\mathbf{n}} \times \Phi_{m}^{(N)}(\mathbf{r})=\mathbf{0} \quad \text { on } S_{N}
$$


It is known, [3], that the fundamental dyadic $\tilde{\Gamma}\left(\mathbf{r}, \mathbf{r}^{\prime}\right)$ and the dyadic $\nabla_{\mathbf{r}^{\prime}} \times \tilde{\Gamma}\left(\mathbf{r}, \mathbf{r}^{\prime}\right)$ have the following expansions:

$$
\begin{aligned}
\tilde{\Gamma}\left(\mathbf{r}, \mathbf{r}^{\prime}\right) & =\sum_{m=0}^{\infty} \frac{\left(i k_{0}\right)^{m}}{m !} \tilde{\zeta}_{m}\left(\mathbf{r}, \mathbf{r}^{\prime}\right), \\
\nabla_{\mathbf{r}^{\prime}} \times \tilde{\Gamma}\left(\mathbf{r}, \mathbf{r}^{\prime}\right) & =\sum_{m=0}^{\infty} \frac{\left(i k_{0}\right)^{m}}{m !} \tilde{\delta}_{m}\left(\mathbf{r}, \mathbf{r}^{\prime}\right)
\end{aligned}
$$

where

$$
\begin{aligned}
& \tilde{\zeta}_{m}\left(\mathbf{r}, \mathbf{r}^{\prime}\right)=-\frac{\left|\mathbf{r}-\mathbf{r}^{\prime}\right|^{m-1}}{4 \pi(m+2)}\left[(m+1) \tilde{I}-\frac{m-1}{\left|\mathbf{r}-\mathbf{r}^{\prime}\right|^{2}}\left(\mathbf{r}-\mathbf{r}^{\prime}\right) \otimes\left(\mathbf{r}-\mathbf{r}^{\prime}\right)\right], \\
& \tilde{\delta}_{m}\left(\mathbf{r}, \mathbf{r}^{\prime}\right)=\frac{1}{4 \pi}(m-1)\left|\mathbf{r}-\mathbf{r}^{\prime}\right|^{m-3}\left(\mathbf{r}-\mathbf{r}^{\prime}\right) \times \tilde{I} .
\end{aligned}
$$

Substituting (4.1), (4.2), (4.7), and (4.8) into (3.5)-(3.7) and equating equal powers of $k_{0}$, the following integral relation among the coefficients $\Phi_{0}^{j}, \Phi_{1}^{j}, \ldots, \Phi_{m}^{j}$ is obtained:

$$
\Phi_{m}^{(0)}(\mathbf{r})=\mathbf{p}(\hat{\mathbf{d}} \cdot \mathbf{r})^{m}+\mathbf{F}_{m}^{(\mathrm{c})}(\mathbf{r})+\mathbf{F}_{m}^{(\mathrm{a})}(\mathbf{r})
$$

where

$$
\begin{aligned}
4 \pi \mathbf{F}_{m}^{(\mathrm{c})}(\mathbf{r})=\beta_{N}^{2} & \frac{\varepsilon_{N}}{\varepsilon_{0}} \sum_{\rho=0}^{m}\left(\begin{array}{c}
m \\
\rho
\end{array}\right) \rho(\rho-1) \int_{S_{N}}\left(\nabla \times \Phi_{\rho-2}^{(N)}\left(\mathbf{r}^{\prime}\right)\right) \cdot\left(\hat{\mathbf{n}} \times \tilde{\zeta}_{m-\rho}\left(\mathbf{r}, \mathbf{r}^{\prime}\right)\right) d s\left(\mathbf{r}^{\prime}\right) \\
& +\frac{1}{\varepsilon_{0}} \sum_{\rho=0}^{m} \sum_{j=1}^{N}\left(\begin{array}{c}
m \\
\rho
\end{array}\right) \rho(\rho-1) \beta_{j}^{2} \varepsilon_{j} \int_{\Omega_{j}}\left(\nabla \times \Phi_{\rho-2}^{(j)}\left(\mathbf{r}^{\prime}\right)\right) \cdot \tilde{\delta}_{m-\rho}\left(\mathbf{r}, \mathbf{r}^{\prime}\right) d v\left(\mathbf{r}^{\prime}\right) \\
& +\sum_{\rho=0}^{m} \sum_{j=1}^{N}\left(\begin{array}{c}
m \\
\rho
\end{array}\right) \rho(\rho-1) \frac{\varepsilon_{j}}{\varepsilon_{0}} \beta_{j} \int_{\Omega_{j}}\left(\nabla \times \Phi_{\rho-2}^{(j)}\left(\mathbf{r}^{\prime}\right)\right) \cdot \tilde{\zeta}_{m-\rho}\left(\mathbf{r}, \mathbf{r}^{\prime}\right) d v\left(\mathbf{r}^{\prime}\right) \\
& +\sum_{\rho=0}^{m} \sum_{j=1}^{N}\left(\begin{array}{c}
m \\
\rho
\end{array}\right) \rho(\rho-1) \frac{\varepsilon_{j}}{\varepsilon_{0}} \beta_{j} \int_{\Omega_{j}} \Phi_{\rho-2}^{(j)}\left(\mathbf{r}^{\prime}\right) \cdot \tilde{\delta}_{m-\rho}\left(\mathbf{r}, \mathbf{r}^{\prime}\right) d v\left(\mathbf{r}^{\prime}\right)
\end{aligned}
$$

and

$$
\begin{aligned}
4 \pi \mathbf{F}_{m}^{(\mathrm{a})}(\mathbf{r})= & \frac{\mu_{0}}{\mu_{N}} \sum_{\rho=0}^{m}\left(\begin{array}{c}
m \\
\rho
\end{array}\right) \int_{S_{N}}\left(\nabla \times \Phi_{\rho}^{(N)}\left(\mathbf{r}^{\prime}\right)\right) \cdot\left(\hat{\mathbf{n}} \times \tilde{\zeta}_{m-\rho}\left(\mathbf{r}, \mathbf{r}^{\prime}\right)\right) d s\left(\mathbf{r}^{\prime}\right) \\
& -\sum_{\rho=0}^{m} \sum_{j=1}^{N}\left(\begin{array}{c}
m \\
\rho
\end{array}\right)\left(1-\frac{\varepsilon_{j}}{\varepsilon_{0}}\right) \rho(\rho-1) \int_{\Omega_{j}} \Phi_{\rho-2}^{(j)}\left(\mathbf{r}^{\prime}\right) \cdot \tilde{\zeta}_{m-\rho}\left(\mathbf{r}, \mathbf{r}^{\prime}\right) d v\left(\mathbf{r}^{\prime}\right) \\
& +\sum_{\rho=0}^{m} \sum_{j=1}^{N}\left(\begin{array}{c}
m \\
\rho
\end{array}\right)\left(\frac{\mu_{0}}{\mu_{j}}-1\right) \int_{\Omega_{j}}\left(\nabla \times \Phi_{\rho}^{(j)}\left(\mathbf{r}^{\prime}\right)\right) \cdot \tilde{\delta}_{m-\rho}\left(\mathbf{r}, \mathbf{r}^{\prime}\right) d v\left(\mathbf{r}^{\prime}\right) .
\end{aligned}
$$

In order to derive the low-frequency expansion for the electric far field pattern $\mathbf{E}_{\infty}(\hat{\mathbf{r}})$, the series (4.2) and

$$
e^{-i k_{0} \hat{\mathbf{r}} \cdot \mathbf{r}^{\prime}}=\sum_{m=0}^{\infty}(-1)^{m} \frac{\left(i k_{0}\right)^{m}}{m !}\left(\hat{\mathbf{r}} \cdot \mathbf{r}^{\prime}\right)^{m}
$$


are substituted into (3.8) to give

$$
\mathbf{E}_{\infty}(\hat{\mathbf{r}})=\mathbf{E}_{\infty}^{(c)}(\hat{\mathbf{r}})+\mathbf{E}_{\infty}^{(a)}(\hat{\mathbf{r}})
$$

where now

$$
\begin{aligned}
4 \pi \mathbf{E}_{\infty}^{(\mathrm{c})}(\hat{\mathbf{r}})=- & \frac{\varepsilon_{N}}{\varepsilon_{0}} \beta_{N}^{2} \sum_{m=0}^{\infty} \frac{\left(i k_{0}\right)^{m+3}}{m !} \sum_{\rho=0}^{m}\left(\begin{array}{c}
m \\
\rho
\end{array}\right)(-1)^{\rho} \int_{S_{N}}\left(\nabla \times \Phi_{m-\rho}^{(N)}\left(\mathbf{r}^{\prime}\right)\right) \\
& \cdot(\hat{\mathbf{n}} \times(\tilde{I}-\hat{\mathbf{r}} \otimes \hat{\mathbf{r}}))\left(\hat{\mathbf{r}} \cdot \mathbf{r}^{\prime}\right)^{\rho} d s\left(\mathbf{r}^{\prime}\right)+\sum_{m=0}^{\infty} \frac{\left(i k_{0}\right)^{m_{i}+4}}{m !} \sum_{\rho=0}^{m}\left(\begin{array}{c}
m \\
\rho
\end{array}\right)(-1)^{\rho} \\
& \times \sum_{j=1}^{N} \frac{\varepsilon_{j}}{\varepsilon_{0}} \beta_{j} \int_{\Omega_{j}}\left(\nabla \times \Phi_{m-\rho}^{(j)}\left(\mathbf{r}^{\prime}\right)\right) \cdot(\tilde{I} \times \hat{\mathbf{r}})\left(\hat{\mathbf{r}} \cdot \mathbf{r}^{\prime}\right)^{\rho} d v\left(\mathbf{r}^{\prime}\right) \\
& +\sum_{m=0}^{\infty} \frac{\left(i k_{0}\right)^{m+4}}{m !} \sum_{\rho=0}^{m}\left(\begin{array}{c}
m \\
\rho
\end{array}\right)(-1)^{\rho} \sum_{j=1}^{N} \frac{\varepsilon_{j}}{\varepsilon_{0}} \beta_{j} \int_{\Omega_{j}} \Phi_{m-\rho}^{(j)}\left(\mathbf{r}^{\prime}\right) \\
& \cdot(\tilde{I} \times \hat{\mathbf{r}})\left(\hat{\mathbf{r}} \cdot \mathbf{r}^{\prime}\right)^{\rho} d v\left(\mathbf{r}^{\prime}\right)-\sum_{m=0}^{\infty} \frac{\left(i k_{0}\right)^{m+3}}{m !} \sum_{\rho=0}^{m}\left(\begin{array}{c}
m \\
\rho
\end{array}\right)(-1)^{\rho} \sum_{j=1}^{N} \frac{\varepsilon_{j}}{\varepsilon_{0}} \beta_{j} \\
& \times \int_{\Omega_{j}}\left(\nabla \times \Phi_{m-\rho}^{(j)}\left(\mathbf{r}^{\prime}\right)\right) \cdot(\tilde{I}-\hat{\mathbf{r}} \otimes \hat{\mathbf{r}})\left(\hat{\mathbf{r}} \cdot \mathbf{r}^{\prime}\right)^{\rho} d v\left(\mathbf{r}^{\prime}\right)
\end{aligned}
$$

and

$$
\begin{aligned}
4 \pi \mathbf{E}_{\infty}^{(\mathrm{a})}(\hat{\mathbf{r}})=- & \frac{\mu_{0}}{\mu_{N}} \sum_{m=0}^{\infty} \frac{\left(i k_{0}\right)^{m+1}}{m !} \sum_{\rho=0}^{m}\left(\begin{array}{c}
m \\
\rho
\end{array}\right)(-1)^{\rho} \int_{S_{N}}\left(\nabla \times \Phi_{m-\rho}^{(N)}\left(\mathbf{r}^{\prime}\right)\right) \\
& \cdot(\hat{\mathbf{n}} \times(\tilde{I}-\hat{\mathbf{r}} \otimes \hat{\mathbf{r}}))\left(\hat{\mathbf{r}} \cdot \mathbf{r}^{\prime}\right)^{\rho} d s\left(\mathbf{r}^{\prime}\right)+\sum_{m=0}^{\infty} \frac{\left(i k_{0}\right)^{m+3}}{m !} \sum_{\rho=0}^{m}\left(\begin{array}{c}
m \\
\rho
\end{array}\right)(-1)^{\rho} \\
& \left.\times \sum_{j=1}^{N}\left(1-\frac{\varepsilon_{j}}{\varepsilon_{0}}\right) \int_{\Omega_{j}} \Phi_{m-\rho}^{(j)}\left(\mathbf{r}^{\prime}\right)\right) \cdot(\tilde{I}-\hat{\mathbf{r}} \otimes \hat{\mathbf{r}})\left(\hat{\mathbf{r}} \cdot \mathbf{r}^{\prime}\right)^{\rho} d v\left(\mathbf{r}^{\prime}\right) \\
& +\sum_{m=0}^{\infty} \frac{\left(i k_{0}\right)^{m+2}}{m !} \sum_{\rho=0}^{m}\left(\begin{array}{c}
m \\
\rho
\end{array}\right)(-1)^{\rho} \sum_{j=1}^{N}\left(\frac{\mu_{0}}{\mu_{j}}-1\right) \\
& \times \int_{\Omega_{j}}\left(\nabla \times \Phi_{m-\rho}^{(j)}\left(\mathbf{r}^{\prime}\right)\right) \cdot(\tilde{I} \times \hat{\mathbf{r}})\left(\hat{\mathbf{r}} \cdot \mathbf{r}^{\prime}\right)^{\rho} d v\left(\mathbf{r}^{\prime}\right) .
\end{aligned}
$$

In order to construct the leading-term approximation as $k_{0} \rightarrow 0$, we first note that the low frequency coefficients $\Phi_{0}^{(j)}$ correspond to $k_{0}=0$ and, in view of (2.7), (2.12), to $\gamma_{j}=0$. Consequently, $\Phi_{0}^{(j)}$ is a solution of the electrostatic problem and, hence, $\Phi_{0}^{(j)}$ is the gradient of a scalar function; therefore, since the coefficient of $i k_{0}$ contains the term $\nabla \times \Phi_{0}^{(N)}$, it is equal to zero. Moreover, the coefficient of $\left(i k_{0}\right)^{2}$ is

$$
\begin{aligned}
& -\frac{1}{4 \pi} \frac{\mu_{0}}{\mu_{N}} \int_{S_{N}}\left(\nabla \times \Phi_{1}^{(N)}\left(\mathbf{r}^{\prime}\right)\right) \cdot(\hat{\mathbf{n}} \times(\tilde{I}-\hat{\mathbf{r}} \otimes \hat{\mathbf{r}})) d s\left(\mathbf{r}^{\prime}\right) \\
& \quad+\frac{1}{4 \pi} \frac{\mu_{0}}{\mu_{N}} \int_{S_{N}}\left(\nabla \times \Phi_{0}^{(N)}\left(\mathbf{r}^{\prime}\right)\right) \cdot(\hat{\mathbf{n}} \times(\tilde{I}-\hat{\mathbf{r}} \otimes \hat{\mathbf{r}}))\left(\hat{\mathbf{r}} \cdot \mathbf{r}^{\prime}\right) d s\left(\mathbf{r}^{\prime}\right)
\end{aligned}
$$




$$
+\frac{1}{4 \pi} \sum_{j=1}^{N}\left(\frac{\mu_{0}}{\mu_{j}}-1\right) \int_{\Omega_{j}}\left(\nabla \times \Phi_{0}^{(j)}\left(\mathbf{r}^{\prime}\right)\right) \cdot(\tilde{I} \times \hat{\mathbf{r}}) d v\left(\mathbf{r}^{\prime}\right) .
$$

If we consider the low frequency expansions for the magnetic fields

$$
\mathbf{H}_{j}(\mathbf{r})=\sum_{m=0}^{\infty} \frac{\left(i k_{0}\right)^{m}}{m !} \Psi_{m}^{(j)}(\mathbf{r}), \quad \mathbf{r} \in \Omega_{j}, \quad j=0,1, \ldots, N
$$

where $\Psi_{m}^{(j)}$ are independent of $k_{0}$, and substitute (4.19), (4.2) into (2.5), we obtain the relation

$$
\begin{aligned}
\nabla \times \Phi_{m}^{(j)}(\mathbf{r})- & m(m-1) \frac{\varepsilon_{j} \mu_{j}}{\varepsilon_{0} \mu_{0}} \beta_{j}^{2} \nabla \times \Phi_{m-2}^{(j)}(\mathbf{r}) \\
& =m \mu_{j}\left(\varepsilon_{0} \mu_{0}\right)^{-1 / 2} \Psi_{m-1}(\mathbf{r})+m(m-1) \frac{\varepsilon_{j} \mu_{j}}{\varepsilon_{0} \mu_{0}} \beta_{j}^{2} \nabla \times \Phi_{m-2}^{(j)}(\mathbf{r}) .
\end{aligned}
$$

From the relation (4.20), we have that the first integral of (4.18) is a multiple of a surface integral of the form

$$
\int_{S_{N}} \Psi_{0}^{N}\left(\mathbf{r}^{\prime}\right) \cdot \hat{\mathbf{n}} d s\left(\mathbf{r}^{\prime}\right)
$$

which is equal to zero, [3]. The other integrals of (4.18) vanish since they contain $\nabla \times \Phi_{0}^{(j)}(\mathbf{r})$. Therefore, the coefficient of $\left(i k_{0}\right)^{2}$ vanishes and the electric far field pattern is of order $O\left(k_{0}^{3}\right)$ as $k_{0} \rightarrow 0$. In particular, the leading term approximation, as $k_{0} \rightarrow 0$, is

$$
\begin{aligned}
\mathbf{E}_{\infty}(\hat{\mathbf{r}})= & \frac{\left(i k_{0}\right)^{3}}{4 \pi}\left\{-\frac{\mu_{0}}{2 \mu_{N}} \int_{S_{N}}\left(\nabla \times \Phi_{2}^{(N)}\left(\mathbf{r}^{\prime}\right)\right) \cdot(\hat{\mathbf{n}} \times(\tilde{I}-\hat{\mathbf{r}} \otimes \hat{\mathbf{r}})) d s\left(\mathbf{r}^{\prime}\right)\right. \\
& +\frac{\mu_{0}}{\mu_{N}} \int_{S_{N}}\left(\nabla \times \Phi_{1}^{(N)}\left(\mathbf{r}^{\prime}\right)\right) \cdot(\hat{\mathbf{n}} \times(\tilde{I}-\hat{\mathbf{r}} \otimes \hat{\mathbf{r}}))\left(\hat{\mathbf{r}} \cdot \mathbf{r}^{\prime}\right) d s\left(\mathbf{r}^{\prime}\right) \\
& +\sum_{j=1}^{N}\left(1-\frac{\varepsilon_{j}}{\varepsilon_{0}}\right) \int_{\Omega_{j}} \Phi_{0}^{(j)}\left(\mathbf{r}^{\prime}\right) \cdot(\tilde{I}-\hat{\mathbf{r}} \otimes \hat{\mathbf{r}}) d v\left(\mathbf{r}^{\prime}\right) \\
& \left.+\sum_{j=1}^{N}\left(\frac{\mu_{0}}{\mu_{j}}-1\right) \int_{\Omega_{j}}\left(\nabla \times \Phi_{1}^{(j)}\left(\mathbf{r}^{\prime}\right)\right) \cdot(\tilde{I} \times \hat{\mathbf{r}}) d v\left(\mathbf{r}^{\prime}\right)\right\}+O\left(k_{0}^{4}\right), k_{0} \rightarrow 0 .
\end{aligned}
$$

Hence, the leading term approximation as $k_{0} \rightarrow 0$ of the electric far field pattern of order $k_{0}^{3}$ in the low frequency case does not depend on the chirality measures $\beta_{j}$ and has the same form as for the achiral multi-layered scatterer [3].

On the other hand, the $k_{0}^{4}$ coefficient of $\mathbf{E}_{\infty}^{(\mathrm{c})}(\hat{\mathbf{r}})$ is given by

$$
\begin{aligned}
\sum_{j=1}^{N} \frac{\varepsilon_{j}}{\varepsilon_{0}} \beta_{j} \int_{\Omega_{j}} \Phi_{0}^{(j)}\left(\mathbf{r}^{\prime}\right) & \cdot(\tilde{I} \times \hat{\mathbf{r}}) d v\left(\mathbf{r}^{\prime}\right) \\
& -\frac{\varepsilon_{N}}{\varepsilon_{0}} \beta_{N}^{2} \int_{S_{N}}\left(\nabla \times \Phi_{1}^{(N)}\left(\mathbf{r}^{\prime}\right)\right) \cdot(\hat{\mathbf{n}} \times(\tilde{I}-\hat{\mathbf{r}} \otimes \hat{\mathbf{r}})) d s\left(\mathbf{r}^{\prime}\right)
\end{aligned}
$$

which is clearly nonzero. Hence, as far as the appearance of chirality in the leading term approximation as $k_{0} \rightarrow 0$ of the electric far field pattern is concerned, the "chirally significant" exponent of $k_{0}$ is 4 . 
REMARK. The most common approach for using the low-frequency method in electromagnetics consists in working with the electric (or the magnetic) field only: [3], [5], [12], [13]. However, one could work with both the electric and magnetic fields; in our case, the decomposition of $\mathbf{E}$ and $\mathbf{H}$ into suitable Beltrami fields, [4], [16], should then be taken into account.

\section{Special cases.}

Achiral multi-layered scatterer. When the chirality measures $\beta_{j}, j=1,2, \ldots$, $N$ are equal to zero (i.e., when the scatterer is achiral multi-layered), then all the terms involving chirality disappear, and the corresponding results of [3] for an achiral multi-layered scatterer are recovered.

Homogeneous (non-layered) chiral scatterer. When $\beta_{j}=\beta, \varepsilon_{j}=\varepsilon, \mu_{j}=\mu$, $j=1,2, \ldots, N$, and there is no core, then the obstacle $\Omega$ is a non-layered homogeneous penetrable chiral scatterer. The existence and uniqueness of solutions to this problem has been established in [4]. In this case, the analysis of the preceding sections gives the following results:

(a) The total exterior electric field is given by $\mathbf{E}_{0}=\mathbf{E}^{\mathrm{inc}}+\mathbf{E}^{\mathrm{sc}(\mathrm{c})}+\mathbf{E}^{\mathrm{sc}(\mathrm{a})}$ where

$$
\begin{aligned}
& 4 \pi \mathbf{E}^{\mathrm{sc}(\mathrm{c})}(\mathbf{r})=-\frac{\varepsilon}{\varepsilon_{0}} k_{0}^{2} \beta\left[\beta \int_{\Omega}\left(\nabla \times \mathbf{E}\left(\mathbf{r}^{\prime}\right)\right) \cdot\left(\nabla_{\mathbf{r}^{\prime}} \times \tilde{\Gamma}\left(\mathbf{r}, \mathbf{r}^{\prime}\right)\right) d v\left(\mathbf{r}^{\prime}\right)\right. \\
& +\int_{\Omega}\left(\nabla \times \mathbf{E}\left(\mathbf{r}^{\prime}\right) \cdot \tilde{\Gamma}\left(\mathbf{r}, \mathbf{r}^{\prime}\right) d v\left(\mathbf{r}^{\prime}\right)+\int_{\Omega} \mathbf{E}\left(\mathbf{r}^{\prime}\right) \cdot\left(\nabla_{\mathbf{r}^{\prime}} \times \tilde{\Gamma}\left(\mathbf{r}, \mathbf{r}^{\prime}\right)\right) d v\left(\mathbf{r}^{\prime}\right)\right] \\
& \begin{aligned}
4 \pi \mathbf{E}^{\mathrm{sc}(\mathrm{a})}(\mathbf{r})=k_{0}^{2}\left(1-\frac{\varepsilon}{\varepsilon_{0}}\right) \int_{\Omega} \mathbf{E}\left(\mathbf{r}^{\prime}\right) \cdot \tilde{\Gamma}\left(\mathbf{r}, \mathbf{r}^{\prime}\right) d v\left(\mathbf{r}^{\prime}\right) \\
+\left(\frac{\mu_{0}}{\mu}-1\right) \int_{\Omega}\left(\nabla \times \mathbf{E}\left(\mathbf{r}^{\prime}\right)\right) \cdot\left(\nabla_{\mathbf{r}^{\prime}} \times \tilde{\Gamma}\left(\mathbf{r}, \mathbf{r}^{\prime}\right)\right) d v\left(\mathbf{r}^{\prime}\right)
\end{aligned}
\end{aligned}
$$

and $\mathbf{E}\left(\mathbf{r}^{\prime}\right)$ is the electric field in $\Omega$.

(b) The electric far field pattern is given by $\mathbf{E}_{\infty}=\mathbf{E}_{\infty}^{(\mathrm{c})}+\mathbf{E}_{\infty}^{(\mathrm{a})}$ where

$$
\begin{aligned}
& \text { (5.3) } 4 \pi \mathbf{E}_{\infty}^{(\mathrm{c})}(\mathbf{r})=\frac{\varepsilon}{\varepsilon_{0}} k_{0}^{3} \beta\left[k_{0} \beta \int_{\Omega}\left(\nabla \times \mathbf{E}\left(\mathbf{r}^{\prime}\right)\right) \cdot(\tilde{I} \times \hat{\mathbf{r}}) e^{-i k_{0} \hat{\mathbf{r}} \cdot \mathbf{r}^{\prime}} d v\left(\mathbf{r}^{\prime}\right)\right. \\
& \left.+i \int_{\Omega}\left(\nabla \times \mathbf{E}\left(\mathbf{r}^{\prime}\right)\right) \cdot(\tilde{I}-\hat{\mathbf{r}} \otimes \hat{\mathbf{r}}) e^{-i k_{0} \hat{\mathbf{r}} \cdot \mathbf{r}^{\prime}} d v\left(\mathbf{r}^{\prime}\right)+k_{0} \int_{\Omega} \mathbf{E}\left(\mathbf{r}^{\prime}\right) \cdot(\tilde{I} \times \hat{\mathbf{r}}) e^{-i k_{0} \hat{\mathbf{r}} \cdot \mathbf{r}^{\prime}} d v\left(\mathbf{r}^{\prime}\right)\right],
\end{aligned}
$$

$$
\begin{aligned}
4 \pi \mathbf{E}_{\infty}^{(\mathrm{a})}(\mathbf{r})=-k_{0}^{2}\left[i k_{0}(\right. & \left.1-\frac{\varepsilon}{\varepsilon_{0}}\right) \int_{\Omega} \mathbf{E}\left(\mathbf{r}^{\prime}\right) \cdot(\tilde{I}-\hat{\mathbf{r}} \otimes \hat{\mathbf{r}}) e^{-i k_{0} \hat{\mathbf{r}} \cdot \mathbf{r}^{\prime}} d v\left(\mathbf{r}^{\prime}\right) \\
& \left.+\left(\frac{\mu_{0}}{\mu}-1\right) \int_{\Omega}\left(\nabla \times \mathbf{E}\left(\mathbf{r}^{\prime}\right)\right) \cdot(\tilde{I} \times \hat{\mathbf{r}}) e^{-i k_{0} \hat{\mathbf{r}} \cdot \mathbf{r}^{\prime}} d v\left(\mathbf{r}^{\prime}\right)\right] .
\end{aligned}
$$

This expression is completely analogous to the one given in [17] after elimination of the terms containing the magnetic field there.

(c) The low-frequency coefficients are given by (4.11) where 
$(5.5)$

$$
\begin{array}{r}
4 \pi \mathbf{F}_{m}^{(c)}(\mathbf{r})=\frac{\varepsilon}{\varepsilon_{0}} \beta \sum_{\rho=0}^{m}\left(\begin{array}{c}
m \\
\rho
\end{array}\right) \rho(\rho-1)\left[\beta \int_{\Omega}\left(\nabla \times \Phi_{\rho-2}\left(\mathbf{r}^{\prime}\right)\right) \cdot \tilde{\delta}_{m-\rho}\left(\mathbf{r}, \mathbf{r}^{\prime}\right) d v\left(\mathbf{r}^{\prime}\right)\right. \\
\left.+\int_{\Omega}\left(\nabla \times \Phi_{\rho-2}\left(\mathbf{r}^{\prime}\right)\right) \cdot \tilde{\zeta}_{m-\rho}\left(\mathbf{r}, \mathbf{r}^{\prime}\right) d v\left(\mathbf{r}^{\prime}\right)+\int_{\Omega} \Phi_{\rho-2}\left(\mathbf{r}^{\prime}\right) \cdot \tilde{\delta}_{m-\rho}\left(\mathbf{r}, \mathbf{r}^{\prime}\right) d v\left(\mathbf{r}^{\prime}\right)\right] \\
4 \pi \mathbf{F}_{m}^{(a)}(\mathbf{r})=-\sum_{\rho=0}^{m}\left(\begin{array}{c}
m \\
\rho
\end{array}\right)\left[\left(1-\frac{\varepsilon}{\varepsilon_{0}}\right) \rho(\rho-1) \int_{\Omega} \Phi_{\rho-2}\left(\mathbf{r}^{\prime}\right) \cdot \tilde{\zeta}_{m-\rho}\left(\mathbf{r}, \mathbf{r}^{\prime}\right) d v\left(\mathbf{r}^{\prime}\right)\right. \\
\left.\quad-\left(\frac{\mu_{0}}{\mu}-1\right) \int_{\Omega}\left(\nabla \times \Phi_{\rho}\left(\mathbf{r}^{\prime}\right)\right) \cdot \tilde{\delta}_{m-\rho}\left(\mathbf{r}, \mathbf{r}^{\prime}\right) d v\left(\mathbf{r}^{\prime}\right)\right]
\end{array}
$$

(d) In the low frequency expansion for the electric far field pattern, we have

$$
\begin{aligned}
4 \pi \mathbf{E}_{\infty}^{(c)}(\mathbf{r})=\frac{\varepsilon}{\varepsilon_{0}} & \beta^{2} \sum_{m=0}^{\infty} \frac{\left(i k_{0}\right)^{m+4}}{m !} \sum_{\rho=0}^{m}\left(\begin{array}{c}
m \\
\rho
\end{array}\right)(-1)^{\rho} \int_{\Omega}\left(\nabla \times \Phi_{m-\rho}\left(\mathbf{r}^{\prime}\right)\right) \\
& \cdot(\tilde{I} \times \hat{\mathbf{r}})\left(\hat{\mathbf{r}} \cdot \mathbf{r}^{\prime}\right)^{\rho} d v\left(\mathbf{r}^{\prime}\right)+\frac{\varepsilon}{\varepsilon_{0}} \beta \sum_{m=0}^{\infty} \frac{\left(i k_{0}\right)^{m+4}}{m !} \sum_{\rho=0}^{m}\left(\begin{array}{c}
m \\
\rho
\end{array}\right)(-1)^{\rho} \\
& \times \int_{\Omega} \Phi_{m-\rho}\left(\mathbf{r}^{\prime}\right) \cdot(\tilde{I} \times \hat{\mathbf{r}})\left(\hat{\mathbf{r}} \cdot \mathbf{r}^{\prime}\right)^{\rho} d v\left(\mathbf{r}^{\prime}\right)-\frac{\varepsilon}{\varepsilon_{0}} \beta \sum_{m=0}^{\infty} \frac{\left(i k_{0}\right)^{m+3}}{m !} \\
& \times \sum_{\rho=0}^{m}\left(\begin{array}{c}
m \\
\rho
\end{array}\right)(-1)^{\rho} \int_{\Omega}\left(\nabla \times \Phi_{m-\rho}\left(\mathbf{r}^{\prime}\right)\right) \cdot(\tilde{I}-\hat{\mathbf{r}} \otimes \hat{\mathbf{r}})\left(\hat{\mathbf{r}} \cdot \mathbf{r}^{\prime}\right)^{\rho} d v\left(\mathbf{r}^{\prime}\right)
\end{aligned}
$$

$$
\begin{aligned}
4 \pi \mathbf{E}_{\infty}^{(a)}(\mathbf{r})=( & \left.1-\frac{\varepsilon}{\varepsilon_{0}}\right) \sum_{m=0}^{\infty} \frac{\left(i k_{0}\right)^{m+3}}{m !} \sum_{\rho=0}^{m}\left(\begin{array}{c}
m \\
\rho
\end{array}\right)(-1)^{\rho} \int_{\Omega} \Phi_{m-\rho}\left(\mathbf{r}^{\prime}\right) \\
& \cdot(\tilde{I}-\hat{\mathbf{r}} \otimes \hat{\mathbf{r}})\left(\hat{\mathbf{r}} \cdot \mathbf{r}^{\prime}\right)^{\rho} d v\left(\mathbf{r}^{\prime}\right)+\left(\frac{\mu_{0}}{\mu}-1\right) \sum_{m=0}^{\infty} \frac{\left(i k_{0}\right)^{m+2}}{m !} \sum_{\rho=0}^{m}\left(\begin{array}{c}
m \\
\rho
\end{array}\right)(-1)^{\rho} \\
& \times \int_{\Omega}\left(\nabla \times \Phi_{m-\rho}\left(\mathbf{r}^{\prime}\right)\right) \cdot(\tilde{I} \times \hat{\mathbf{r}})\left(\hat{\mathbf{r}} \cdot \mathbf{r}^{\prime}\right)^{\rho} d v\left(\mathbf{r}^{\prime}\right) .
\end{aligned}
$$

6. Appendix. In this Appendix, we establish that the electric fields may be expanded into convergent power series of $k_{0}$. For simplicity, and without loss of generality, we consider that $\Omega$ is a non-layered homogeneous chiral scatterer (of electric permittivity $\varepsilon$, magnetic permeability $\mu$, and chirality measure $\beta$ ) immersed in free space $\Omega_{0}$.

Let $\eta$ be the intrinsic impedance $(\eta=\sqrt{\mu / \varepsilon})$ of $\Omega$, and let $k^{2}=\omega^{2} \varepsilon \mu$. Let us note that by the definition of $k_{0}$ :

$$
k^{2}=\frac{\varepsilon \mu}{\varepsilon_{0} \mu_{0}} k_{0}^{2} .
$$

Recall the well-known Bohren transform [16]

$$
\mathbf{E}=\mathbf{Q}_{L}-i \eta \mathbf{Q}_{R}, \quad \mathbf{H}=\frac{1}{i \eta} \mathbf{Q}_{L}+\mathbf{Q}_{R},
$$


of the electric and magnetic fields $\mathbf{E}$ and $\mathbf{H}$ in $\Omega$ into left-handed and right-handed Beltrami fields $\mathbf{Q}_{L}$ and $\mathbf{Q}_{R}$, respectively, satisfying

$$
\nabla \times \mathbf{Q}_{L}=\gamma_{L} \mathbf{Q}_{L}, \quad \nabla \times \mathbf{Q}_{R}=-\gamma_{R} \mathbf{Q}_{R}
$$

where the wave numbers corresponding to $\mathbf{Q}_{L}$ and $\mathbf{Q}_{R}$ are given by

$$
\gamma_{L}=k(1-k \beta)^{-1}, \quad \gamma_{R}=k(1+k \beta)^{-1} .
$$

Since $\mathbf{E}$ and $\mathbf{H}$ are divergence free, an immediate consequence of (6.3) is

$$
\Delta \mathbf{Q}_{L}+\gamma_{L}^{2} \mathbf{Q}_{L}=\mathbf{0}, \quad \Delta \mathbf{Q}_{R}+\gamma_{R}^{2} \mathbf{Q}_{R}=\mathbf{0}, \text { in } \Omega
$$

It is known, see e.g., [21], that $\mathbf{Q}_{L}$ depends analytically on $\gamma_{L}$, for $\gamma_{L} \neq 0$ (and $\mathbf{Q}_{R}$ on $\gamma_{R}$ ). Since, by (6.4), $\gamma_{L}$ and $\gamma_{R}$ are rational functions of $k, \mathbf{Q}_{L}$ and $\mathbf{Q}_{R}$ depend analytically on $k$; hence, by (6.2), $\mathbf{E}$ and $\mathbf{H}$ are both analytic in $k$, and in view of (6.1) in $k_{0}$. Thus, we have established the desired property.

Acknowledgment. The authors thank Dr. W. S. Weiglhofer (University of Glasgow, U.K.) for constructive discussions. In addition, they thank the referees for substantial comments that led to the present form of the paper. They also acknowledge partial financial support from the General Secretariat of Research and Technology of the Ministry of Development (Greece), within the PENED 1994 programme.

\section{REFERENCES}

[1] H. Ammari, M. Laouadi, AND J.-C. NÉdÉLec, Low frequency behavior of solutions to electromagnetic scattering problems in chiral media, SIAM J. Appl. Math., 58 (1998), pp. 1022-1042.

[2] C. AthHANASIADIS, The multi-layered ellipsoid with a soft core in the presence of a low-frequency acoustic wave, Quart. J. Mech. Appl. Math., 47 (1994), pp. 441-459.

[3] — Low-frequency electromagnetic scattering theory for a multi-layered scatterer, Quart. J. Mech. Appl. Math., 44 (1991), pp. 55-67.

[4] C. Athanasiadis AND I. G. STRATIS, Electromagnetic scattering by a chiral object, IMA J. Appl. Math., 58 (1997), pp. 83-91.

[5] - The conductive problem for Maxwell's equations at low frequencies, Appl. Math. Lett., 10 (1997), pp. 101-105.

[6] - On the scattering of electromagnetic waves by a piecewise homogeneous chiral obstacle, in Mathematical Methods in Scattering Theory and Biomedical Technology, G. Dassios, et al., ed., Addison-Wesley-Longman, London, 1998, pp. 173-185.

[7] D. Colton And R. KRess, Integral Equation Methods in Scattering Theory, Wiley, New York, 1983.

[8] G. DAssios, Low frequency scattering theory for a penetrable body with an impenetrable core, SIAM J. Appl. Math., 42 (1982), pp. 272-280.

[9] G. DAssios AND K. KIRIAKI, The low-frequency theory of elastic wave scattering, Quart. Appl. Math., 42 (1984), pp. 225-248.

[10] D. L. Jaggard, X. Sun, And J. C. Lin, On the chiral Riccati equation, Microwave Opt. Techn. Lett., 5 (1992), pp. 107-112.

[11] D. S. Jones, Low frequency electromagnetic radiation, J. Inst. Math. Appl., 23 (1979), pp. 421-447.

[12] — Scattering by inhomogeneous dielectric particles, Quart. J. Mech. Appl. Math., 38 (1985), pp. 135-155.

[13] R. E. KLEINMAN, Low frequency solution of electromagnetic scattering problems, in Electromagnetic Wave Theory, J. Brown, ed., Pergamon, Oxford, 1967, pp. 891-905.

[14] R. E. KLEINMAN AND B. VAINBERG, Full low-frequency asymptotic expansion for second-order elliptic equations in two dimensions, Math. Meth. Appl. Sci., 17 (1994), pp. 989-1004.

[15] A. LAKHTAKIA, Rayleigh scattering by a bianisotropic ellipsoid in a biisotropic medium, Int. J. Electronics, 6 (1991), pp. 1057-1062. 
[16] - Beltrami Fields in Chiral Media, World Scientific, Singapore, 1994.

[17] A. LAKhtAkia, V. K. VARAdan, AND V. V. VARADAN, Dilute random distribution of small chiral spheres, Appl. Optics, 29 (1990), pp. 3627-3632.

[18] R. PICARD, On the low frequency asymptotics in electromagnetic theory, J. Reine Angew. Math., 354 (1984), pp. 50-73.

[19] A. F. STEvenson, Solution of electromagnetic scattering problems as power series in the ratio (dimension of scatterer)/wavelength, J. Appl. Phys., 24 (1953), pp. 1134-1142.

[20] V. TWERSKY, Multiple scattering of electromagnetic waves by arbitrary configurations, J. Math. Phys., 8 (1967), pp. 589-610.

[21] P. WERNER, On the exterior boundary value problem of perfect refraction for stationary electromagnetic wave fields, J. Math. Anal. Appl., 7 (1963), pp. 348-396. 
